\title{
Saccharide Sensing Using Gold and Silver Nanoparticles-A Review
}

\author{
Kadir Aslan,, ${ }^{1,2}$ Jian Zhang, ${ }^{2}$ Joseph R. Lakowicz, ${ }^{2}$ and Chris D. Geddes ${ }^{1,2,3}$
}

Received January 14, 2004; revised February 18, 2004; accepted February 18, 2004

\begin{abstract}
We review new methodologies for glucose sensing from our laboratories based on the specific biological interactions between Con A, dextran-coated gold nanoparticles and glucose, and the interactions between dextran, glucose, and boronic-acid capped silver nanoparticles in solution. Our new approaches promise new tunable glucose sensing platforms. Dextran-coated gold nanoparticles were aggregated with the addition of Con A resulting in increase an in absorbance of nanoparticles at 650 $\mathrm{nm}$, where the post-addition of glucose caused the dissociation of the aggregates and thus a decrease in the absorbance at $650 \mathrm{~nm}$. The interaction of glucose and dextran with boronic acid-capped silver nanoparticles in solution resulted in enhanced luminescence intensity cumulatively due to surfaceenhanced fluorescence and the decrease in absorbance at $400 \mathrm{~nm}$, with an increase in absorbance at $640 \mathrm{~nm}$. Lifetime measurements were used to distinguish the contribution from the surface-enhanced fluorescence. TEM was employed to assess the aggregation of nanoparticles.
\end{abstract}

KEY WORDS: Gold colloids; silver colloids; glucose sensing; nanosensors; monosaccharide; polysaccharide; plasmons; surface plasmon resonance.

\section{INTRODUCTION}

Diabetes is well-known to result in long-term health disorders including cardiovascular disease and blindness. One of the major challenges in the management of diabetes is the monitoring of glucose concentrations. Despite intensive efforts [1-5], no method is currently available for the continuous non-invasive monitoring of blood glucose.

Metallic nanostructures have been studied extensively and are emerging as important colorimetric reporters due to their high extinction coefficients, which are typically several orders of magnitude larger than those of organic dyes [6]. In particular, nanostructures made from

\footnotetext{
${ }^{1}$ Institute of Fluorescence and Center for Fluorescence Spectroscopy, Medical Biotechnology Center, University of Maryland Biotechnology Institute, 725 West Lombard Street, Baltimore, Maryland, 21201.

2 Center for Fluorescence Spectroscopy, Department of Biochemistry and Molecular Biology, University of Maryland School of Medicine, 725 West Lombard Street, Baltimore, Maryland, 21201.

3 To whom correspondence should be addressed; E-mail: cfs@ cfs.umbi.umd.edu
}

the noble metals, such as those of silver or gold, with their associated strong plasmon resonance, have generated great interest [6-13]. Gold nanoparticles display plasmon absorption bands that depend on their size and shape [7-9]. Nanoparticle aggregation results in further color changes of gold nanoparticle solutions due to mutually induced dipoles that depend on interparticle distance and aggregate size [10-13]. The fact that the plasmon resonance is a sensitive function of nanostructure geometry, coupled with synthetic advances that allow for the controlled and systematic variations in nanostructure geometry, is leading to the expanse of this sensing field called "plasmonics." Gold nanoparticle aggregation induced by analytes has

\footnotetext{
ABBREVIATIONS: AEE, 2-(2-aminoethoxy)ethanol; Con A, Concanavalin A; EDC, $N$-3-(Dimethylaminopropyl)- $N$ '-ethylcarbodiimide; Diglyme, 2-methoxyethyl ether; FRET, Fluorescence Resonance Energy Transfer; 16-MHDA, 16-Mercaptohexadecanoic acid; Nanogold, $20 \mathrm{~nm}$ diameter gold colloids; NHS, N-Hydroxy2,5-pyrrolidinedione; PBS, Phosphate buffered saline; SPR, Surface Plasmon Resonance; TEM, Transmission Electron Micrograph; SNBA, Silver nanoparticles capped with thiolated boronic acid groups.
} 

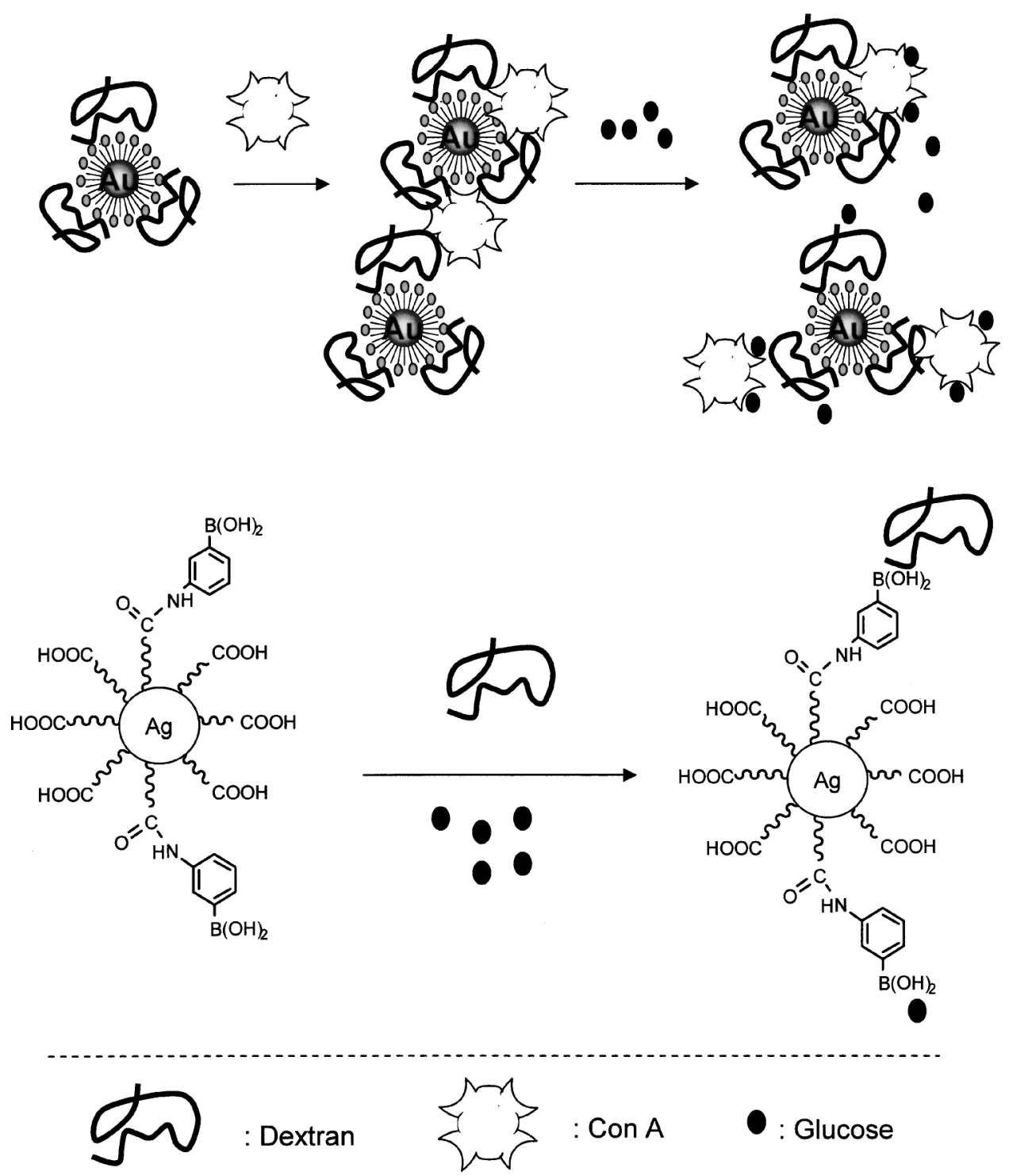

Fig. 1. Glucose sensing mechanism: the dissociation of Con A aggregated dextran-coated gold nanoparticles (Top), interactions of glucose and dextran with silver nanoparticles containing boronic acid group (Bottom).

been demonstrated for DNA [14,15], metal ions [16,17] and antibodies [18]. However, to the best of our knowledge, their use in glucose sensing has not been explored.

In this paper we report the preparation of dextrancoated gold nanoparticles and boronic acid-caped silver nanoparticles, which were tested as glucose sensing platforms. The sensing mechanism using dextran-coated gold nanoparticles is based on the specific interactions between dextran and Con A, and Con A and glucose (Fig. 1), which resulted in change in absorption characteristics of the gold nanoparticles. The interactions of boronic acid-capped silver nanoparticles and glucose and dextran, resulted in aggregation of the nanoparticles, which is accompanied by an enhanced luminescence intensity and change in absorbance at $640 \mathrm{~nm}$ (Fig. 1).

\section{METHODOLOGY}

\section{Materials}

Gold nanoparticle dispersions (monodisperse, $20 \mathrm{~nm}$ average particle diameter), silver nitrate Concanavalin A (Con A from Canavalia ensiformis), dextran (average molecular weight: 3,000, 64,000 (64 K), 170,000 (170 K) and 505,000 $(500 \mathrm{~K})$, glucose hydrogen peroxide, sulfuric acid, sodium phosphate monobasic, sodium 
borohydride, phosphate buffered saline (PBS), absolute ethanol, methanol, (2-(2-aminoethoxy)ethanol (AEE) and $N$-Hydroxy-2,5-pyrrolidinedione (NHS) were obtained from Sigma. 16-Mercaptohexadecanoic acid (16-MHDA) and polyoxyethylene (20) sorbitan monolaurate (Tween 20), epichlorohydrin, 2-methoxyethyl ether (diglyme), $N$-(2-mercaptopropionyl)glycine (tiopronin), 2,2dimethyl-propane-diol, 1,3-dicyclohexylcarbodiimide (DCC), tetrahedrafuran (THF) and nitric acid were obtained from Aldrich. $N$-3-(Dimethylaminopropyl)- $N^{\prime}$ ethyl-carbodiimide (EDC) was obtained from Fluka. All chemicals were used as received.

\section{Buffers and Solutions}

Sodium phosphate monobasic buffer solution was prepared to a $10 \mathrm{mM}$ concentration at $\mathrm{pH} 7$. PBS was dissolved in deionized water and the $\mathrm{pH}$ was adjusted to 7.4. Exact $\mathrm{pH}$ values for buffer solutions were obtained using a Beckman $\mathrm{pH}$ meter. Deionized water
( $>18 \mathrm{M} \Omega / \mathrm{cm}$ ) was used in the preparation of all buffer solutions. All glassware was washed with "piranha solution" (3:7 30\% $\mathrm{H}_{2} \mathrm{O}_{2} / \mathrm{H}_{2} \mathrm{SO}_{4}$ ) prior to use. Solutions of 0.50 $\mathrm{mM}$ 16-MHDA were prepared in degassed ethanol. Tween 20 solutions were prepared in sodium phosphate buffer at $\mathrm{pH} 7$.

\section{Preparation of the Dextran-Coated Gold Nanoparticles}

The immobilization of dextran on gold nanoparticles was performed using the following procedure as described previously [19]; in the first step, gold nanoparticle dispersions were modified with 16-MHDA presenting $-\mathrm{COOH}$ groups in the presence on Tween 20. Then, AEE was reacted with - $\mathrm{COOH}$ groups using carbodiimide chemistry. This step resulted in gold nanoparticles with the $-\mathrm{OH}$ functionality. The $-\mathrm{OH}$ groups were then activated with epicholorohydrin, and dextran was coupled covalently to these groups (Fig. 2-Top) [20]. Dextran-coated
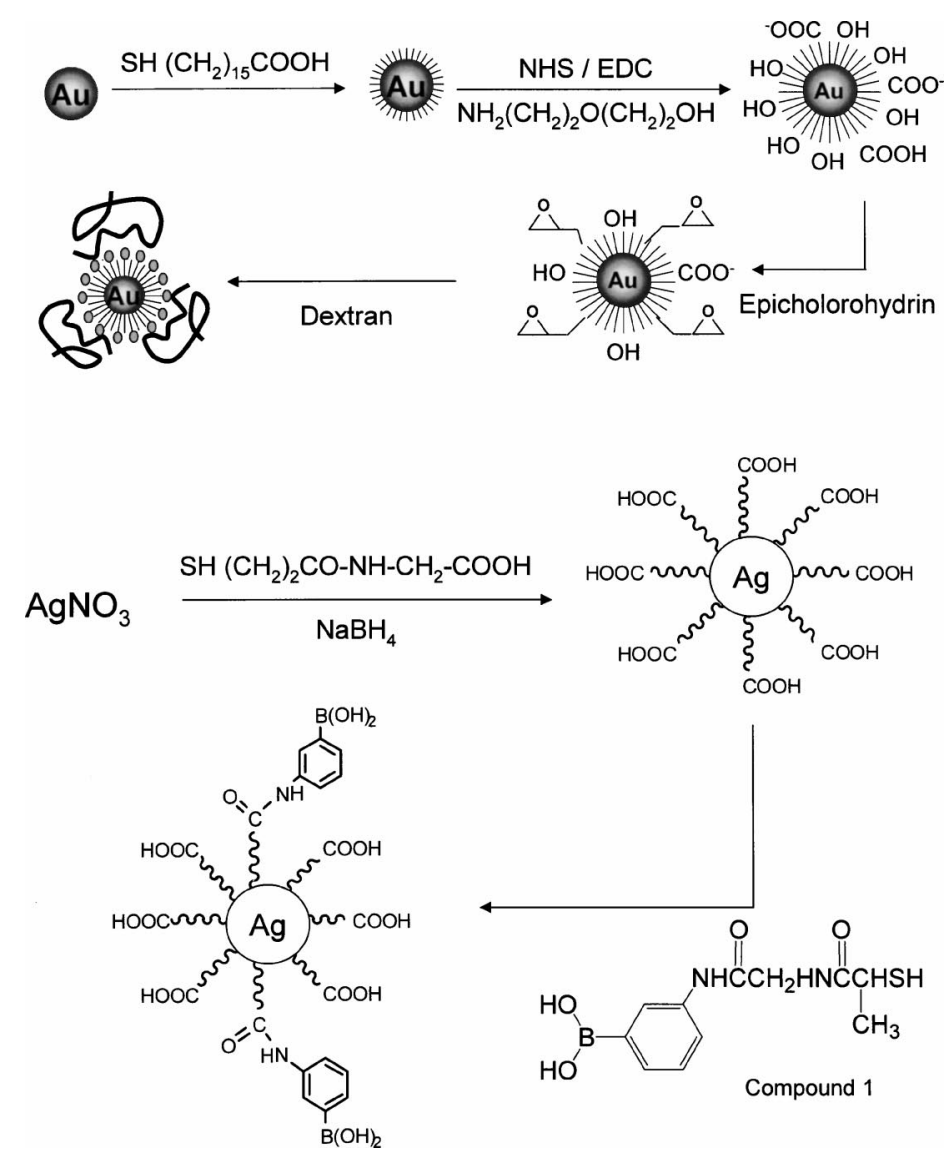

Fig. 2. Synthetic scheme for the preparation of the dextran-coated gold colloids (Top), and the silver nanoparticles with boronic acid groups (Bottom). 


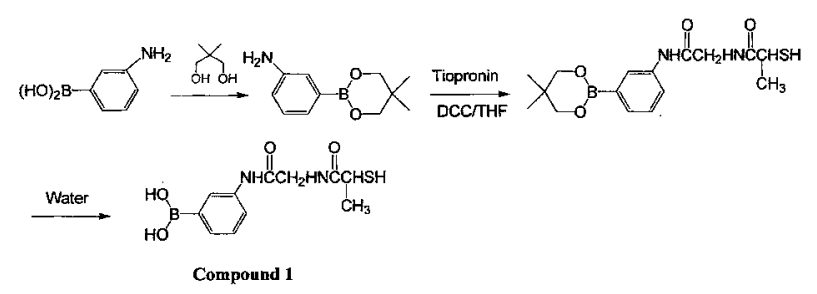

Schme 1. Synthesis of thiolated boronic acid (Compound 1).

gold nanoparticles were purified with extensive centrifugation and resuspension cycles. All solutions with gold nanoparticles were stored in polypropylene tubes to prevent light-induced flocculation [21].

\section{Preparation of Boronic Acid Functionalized Silver Nanoparticles}

Silver nanoparticles containing mixed monolayers of tiopronin and thiolated boronic acid were synthesized according to the procedure described previously (Fig. 2Bottom) [22]. The reaction scheme for the synthesis of thiolated boronic acid is shown in Scheme 1.

In a typical synthesis, silver nitrate is firstly reduced by sodium borohydride in the presence of tiopronin in methanol. This yields silver nanoparticles with the $-\mathrm{COOH}$ functionality and sizes of approximately $1-$ $5 \mathrm{~nm}$. Then, aqueous solutions of thiolated boronic acid and tiopronin-modified silver nanoparticles were stirred for $72 \mathrm{hr}$ at room temperature. Water was removed under vacuum and the residue was washed with methanol to remove uncapped thiolated boronic acid. The mixed monolayer compositions were assessed by ${ }^{1} \mathrm{H}-\mathrm{NMR}$ spectra with a mole ratio of aromatic protons and methyl on the tiopronin [22].

\section{Methods}

All absorption measurements were performed using a Varian UV/VIS 50 Spectrophotometer and Hewlett Packard 8453 spectrophotometer in $1 \mathrm{~cm}$ Quartz cuvettes (Starna). ${ }^{1} \mathrm{H}-\mathrm{NMR}$ spectra were recorded on GE-QE 300 spectrometer. Luminescence spectra were recorded with Cary Eclipse Fluorescence Spectrophotometer. Transmission electron micrographs (TEM) of the nanoparticles were taken with a side-entry Philips electron microscope operated at $120 \mathrm{keV}$. Samples were cast from water solutions onto standard carbon-coated (200-300 §) Formvar films on copper grids (400 mesh), waiting $5 \mathrm{~min}$, and removing excess solution by touching a small piece of filter paper to the edge of the grid. The grid was dried under $\mathrm{N}_{2}$ flow or in air for $30 \mathrm{~min}$.
Organic reactions were monitored by thin-layer chromatography on $0.25 \mathrm{~mm}$ Merck silica gel plates, while Baxter silica gel $60 \AA$ (230-400 mesh ASTM) was used for flash column chromatography.

\section{RESULTS AND DISCUSSION}

\section{Con A-Induced Aggregation of Dextran-Coated Gold Nanoparticles}

For colloidal gold in an aqueous medium, the surface plasmon peak is observed around $520 \mathrm{~nm}$. However, if the colloidal particles aggregate such that the distance between aggregating spheres becomes small compared to their radius, additional resonances will occur at wavelengths longer than those of the individual particles. This results in red-shifting and broadening of the absorption spectra [23]. Since Con A is a multivalent protein (four binding sites are present at $\mathrm{pH} 7$ ), at least two or more dextran-coated gold nanoparticles could be cross-linked due to specific biological interactions between dextran (a biopolymer consisting of glucose sub-units) and Con A. These interactions result in aggregation of the nanoparticles which is monitored by the increase in absorbance at arbitrary wavelengths longer than $600 \mathrm{~nm}$ (Fig. 3-Top) [23]. We subsequently monitored the aggregation of dextrancoated gold nanoparticles at $650 \mathrm{~nm}$ with respect to time, since the change in absorbance of aggregating nanoparticles was the largest at this wavelength. Figure 3-Bottom shows the time dependent change in absorbance at $650 \mathrm{~nm}$ for $500 \mathrm{~K}$ dextran coated gold nanoparticles after the addition of $4-110 \mu \mathrm{M}$ of Con A. As the amount of Con $\mathrm{A}$ is increased, the change in absorbance at $650 \mathrm{~nm}$ of dextran-coated gold nanoparticles is also increased. We note that when the concentration of Con A was larger than $75 \mu \mathrm{M}$, the dextran coated gold nanoparticles completely aggregated and the solution became turbid and thus, not practical for sensing applications.

Figure 4-Top shows the maximum value $(95 \%)$ of change in absorbance at $650 \mathrm{~nm}$ for dextran coated gold nanoparticles versus the concentration of Con A used. The largest change in absorbance was observed with the $500 \mathrm{~K}$ dextran coated gold nanoparticles. When the molecular weight of the dextran is decreased to $170 \mathrm{~K}$ and subsequently to $64 \mathrm{~K}$, smaller changes in absorbance were typically observed. This clearly shows that the extent of aggregation of the nanoparticles depends on the concentration of Con A, as well as the molecular weight of dextran immobilized onto the surface of the gold nanoparticles. Our explanation for this observation is the availability of dextran on the surface of the gold nanoparticles for Con A binding, that is, since the molecular weight of tetrameric 

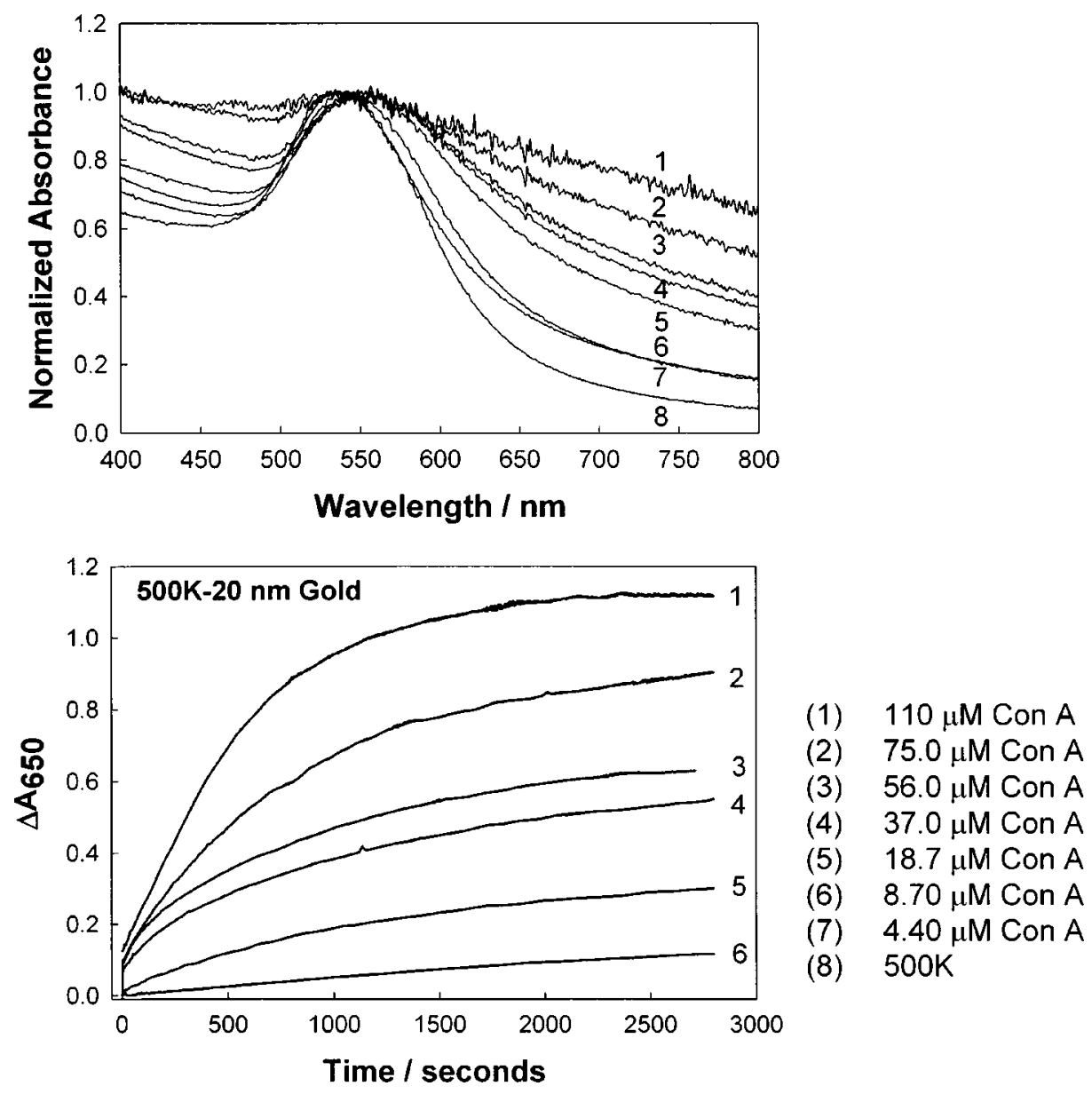

Fig. 3. Normalized absorbance spectra of dextran-coated gold nanoparticles (500K) after the addition of Con A (Top), time-dependent change in absorbance at $650 \mathrm{~nm}$ for $500 \mathrm{~K}$ dextran coated gold nanoparticles (Bottom).

protein Con $\mathrm{A}$ (at pH 7) is approximately 104,000 daltons, it is hypothesized that the extent of interaction between Con A and dextran is limited by the size of the dextran. Thus, the probability of cross-linking of the dextrancoated gold nanoparticles by Con A molecules is higher, when high molecular weight dextran (505,000 daltons, denoted as $500 \mathrm{~K}$ ) is used. Logically, when the size of dextran is decreased; the probability of cross-linking is also decreased.

Figure 4-Bottom shows the TEM image of aggregates of dextran-coated gold nanoparticles $(500 \mathrm{~K})$ before and after the addition of $110 \mu \mathrm{M}$ Con A. It is observed that dextran-coated gold nanoparticles are separated from each other before the addition of Con A, and they came closer after the addition of Con A, presenting an evidence that Con A interacts with dextran on the surface of different nanoparticles, resulting in Con A-dextran coated gold nanoparticle sensing aggregates. However, it should be noted that as a result of the subjective nature of the
TEM and possible artifacts resulting from evaporation of the solvent and interactions between the nanoparticles and the TEM substrate, measurement of exact sizes of the aggregates may prove unreliable by TEM. Thus, in this study, TEM was used to verify and provide qualitative information about the hypothesis on protein-induced aggregation of the nanoparticles, rather than obtaining quantitative information on the size of the aggregates.

\section{Sensing of Glucose by the Dissociation of Con A/Dextran Nanogold Aggregates}

Figure 5-Top shows the time-dependent change in absorbance at $650 \mathrm{~nm}$ of dextran-coated gold nanoparticle aggregates after the addition of glucose. The addition of glucose is expected to cause the dissociation of Con A due to the competition of glucose units of dextran with the glucose additionally added. Given that, the dextran-coated gold nanoparticles completely aggregated 

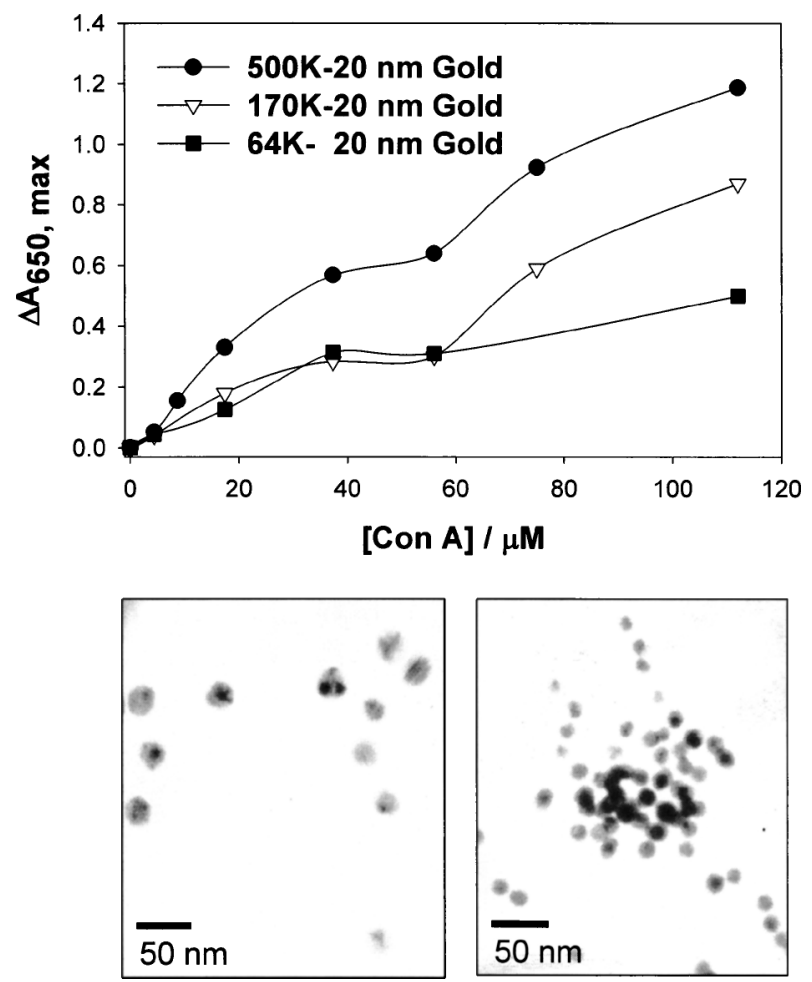

Fig. 4. Maximum change in absorbance at $650 \mathrm{~nm}$ for dextran coated gold nanoparticles versus the concentration of Con A used (Top). TEM images of $500 \mathrm{~K}$ dextran coated gold nanoparticles before (Bottom) and after the addition of $110 \mu \mathrm{M}$ Con $\mathrm{A}(\mathbf{C})$.

when the concentration of Con A was larger than $75 \mu \mathrm{M}$, the addition of glucose to the highly aggregated nanoparticles $(110 \mu \mathrm{M}$ Con $\mathrm{A})$ did not cause any significant change in the absorbance even when the amount of glucose was perversely increased. However, we did observe a significant amount of change in absorbance when the aggregation of the dextran-coated gold nanoparticles were moderate (with $18.7 \mu \mathrm{M}$ Con A). This is attributed to the fact that when the Con A concentration was smaller than $75 \mu \mathrm{M}$ most of the sensing aggregates remained in suspension, thus allowing the competitive dissociation of aggregates to proceed.

Figure 5-Bottom shows the cumulative response of $500 \mathrm{~K}$ dextran-coated gold nanoparticles aggregated with Con A (concentration ranging from $4 \mu \mathrm{M}$ to $75 \mu \mathrm{M}$ ), towards glucose. The dissociation of the dextran-coated gold nanoparticle/Con A aggregates with glucose were however insignificant when the Con A concentration was too low $(4 \mu \mathrm{M})$ or too large (above $75 \mu \mathrm{M}$ ). Since the change in absorbance was very small with $4 \mu \mathrm{M}$ Con $\mathrm{A}$, the recovery of the signal $\left(-\Delta \mathrm{A}_{650}\right)$ was also small. Larger concentrations of Con A (above $75 \mu \mathrm{M}$ ) caused the aggregation of almost all available dextran-coated gold
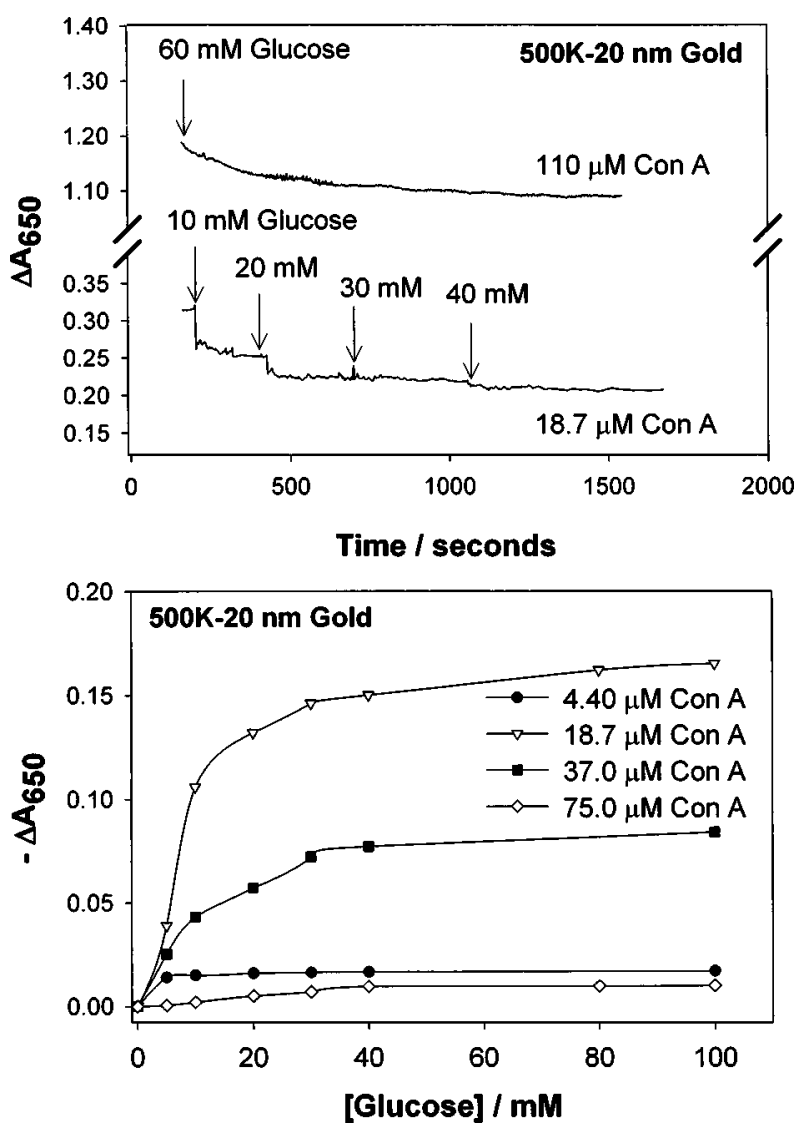

Fig. 5. Time-dependent change in absorbance at $650 \mathrm{~nm}$ for $500 \mathrm{~K}$ dextran coated gold nanoparticles after the addition of glucose (Top), and cumulative change in $650 \mathrm{~nm}$ absorbance for $500 \mathrm{~K}$ dextran coated gold nanoparticles versus the concentration of glucose (Bottom).

nanoparticles in solution, and thus, the amount of glucose added to the sensing aggregates $(0-100 \mathrm{mM})$ was not enough to cause competitive dissociation. However, better glucose responses were observed when the dextrancoated gold nanoparticles were aggregated with between 18.7 and $37 \mu \mathrm{M}$ of Con A. The change in absorbance reached a maximum with the addition of approximately $40 \mathrm{mM}$ glucose, indicating the glucose dynamic sensing range.

We note that while study is investigatory in nature, it has important implications in terms of employing noble metals and biomolecules for non-invasive glucose monitoring. We also predict that the sensitivity of sensing of glucose with dextran-coated gold nanoparticles can be improved by modifying the current preparation method: using alkane thiols containing ethylene glycol (EG) groups instead of simple long chain alkane thiols. It is well documented that the alkane thiols with EG groups resist the non-specific adsorption of proteins onto metal surfaces and therefore could reduce the aggregation of nanoparti- 
cles due to non-specific interactions of Con A with the surface of the gold nanoparticles. Further findings by our laboratories will be reported in due course.

\section{Silver Nanoparticles Containing Boronic Acid Moiety}

\section{Preparation of Thiolated Boronic Acid and Its} Interactions with Glucose in Solution

The thiolated boronic acid (compound 1, Fig. 2Bottom) was prepared by condensation from 3aminophenyl boronic acid and tiopronin using DCC as the condensation reagent as reported earlier [22]. In order to avoid the reaction of boronic acid with DCC, the boronic acid had to be protected by 2,2-dimethyl-propanediol before condensation, and then was de-protected in water. The final product was dissolved in alcohol and water. The thiolated boronic acid displayes an absorbance maximum at $296 \mathrm{~nm}$ in an aqueous solution and a fluorescence peak at $377 \mathrm{~nm}$ when excited at its absorbance maximum (Fig. 6-Top). It could be coupled with either a monosaccharide (glucose) or polysaccharide (Dextran) forming the respective boronate ester, where the luminescence intensities increased by up to $40-50 \%$, when the concentration of carbohydrate unit in solution was at least 10 times higher than that of boronic acid. Their association constants with saccharides were estimated to be $3.3 \times$ $10^{3}$ and $4.9 \times 10^{3} \mathrm{M}^{-1}$ based on the relation between the luminescence intensity and concentration of carbohydrate $[24,25]$.

\section{Interactions of Glucose and Dextran with Silver Nanoparticles Containing the Boronic Acid Moiety}

Figure 6-Bottom shows the luminescence spectra of silver nanoparticles capped with thiolated boronic acid groups (SNBA) before and after the addition of glucose at $\mathrm{pH} 5$ in water. The luminescence intensity of SNBA is increased as glucose interacted with the boronic acid groups on the surface of the silver nanoparticles.

Figure 7-Top shows the absorption spectra of $60 \mathrm{nM}$ SNBA in the presence of increasing amounts of dextran. As the amount of dextran is increased the surface plasmon peak at $397 \mathrm{~nm}$ is decreased, accompanied by an increase in a plasmon peak at longer wavelengths $(640 \mathrm{~nm})$. Figure 7B shows the change in these peaks as well as the ratio of both peaks. We hypothesize that the decrease in absorbance at $397 \mathrm{~nm}$ and the increase in $640 \mathrm{~nm}$ indicate that the silver nanoparticles aggregate in a linear fashion around the dextran chain due to the interactions of boronic acid groups with the glucose sub-units on dextran chain. This hypothesis was also confirmed by
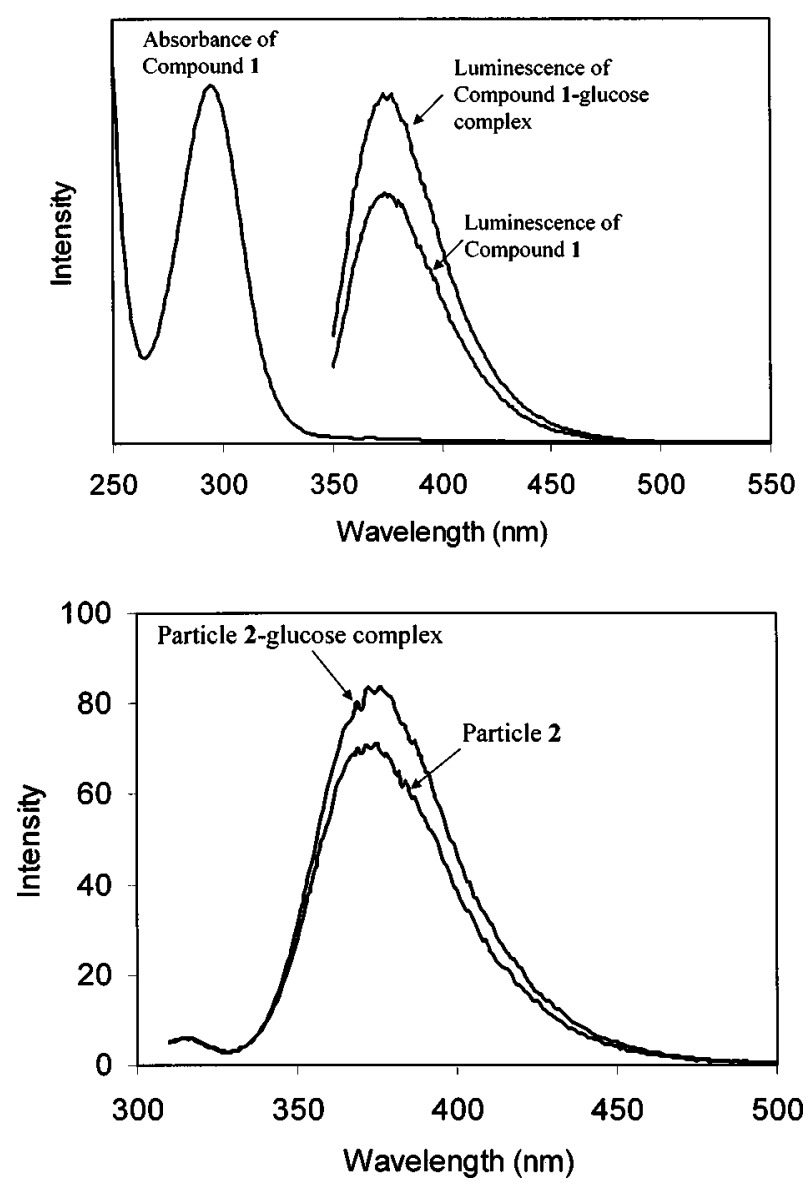

Fig. 6. Absorbance and luminescence spectrum of compound $\mathbf{1}$ in water (Top), and luminescence spectrum of SNBA before and after coupling with glucose (Bottom), $\lambda_{\mathrm{ex}}=300 \mathrm{~nm}$.

TEM pictures of SNBA and the aggregates of SNBA/ dextran, which are shown in Fig. 8. As indicated earlier, due to subjective nature of TEM, these results are used only as a visual evidence for the aggregation of the nanoparticles.

Figure 9-Top shows the luminescence spectra of SNBA in the presence of increasing amounts of dextran. The interaction of boronic acid groups and dextran caused an increase in luminescence. This is attributed to the aggregation of the SNBA. When the nanoparticles come in close proximity to one another, the emissions from the luminescent species is thought to increase due to the local high-electric fields of the silver nanoparticles and a modification in the radiative decay rate (surfaceenhanced fluorescence), where the lifetimes of these species are reduced. In addition to this, the absorbance at the surface plasmon peak $(400 \mathrm{~nm})$ decreases due to the aggregation of the nanoparticles, the emissions from the luminescent species absorbed by the nanoparticles 

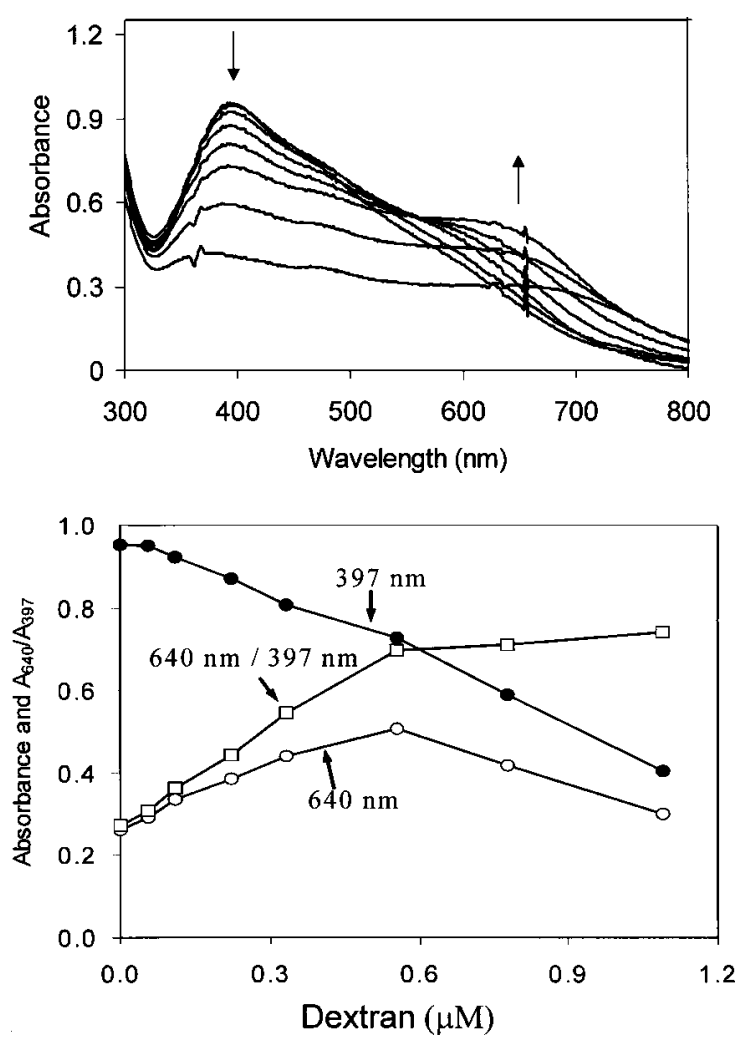

Fig. 7. Absorbance spectra of the $60 \mathrm{nM}$ SNBA before and after the addition of dextran after 2 hours of coupling in water (Top), absorbance at 397 and $640 \mathrm{~nm}$ and absorbance ratio of $640 \mathrm{~nm} / 397 \mathrm{~nm}$ vs. concentration of dextran in solution (Bottom).

decreases resulting in larger intensities (i.e. reduced innerfilter effect). Figure 9-Bottom shows the enhancement factor $\left.\Delta I-I_{0}\right) / I_{0}$ (calculated from luminescence intensities $(I)$ from Figure 9-Bottom) versus the concentra- tion of dextran used. The luminescence intensity is increased up to two-folds as the concentration of dextran is increased.

It is informative to consider the decay times to distinguish the contribution from surface-enhanced fluorescence [26-28]. The intensity decays were measured using the well-known Time-Correlated Single Photon Timing Technique [29] and analyzed in terms of the multiexponential model:

$$
I(t)=\sum_{i} \alpha_{i} \exp (-t / \tau)
$$

where $\alpha_{I}$ are the amplitudes and $\tau_{I}$ the decay times, $\Sigma \alpha_{I}=$ 1.0. The fractional contribution of each component to the steady-state intensity is given by:

$$
f_{i}=\frac{\alpha_{i} \tau_{i}}{\sum_{i} \alpha_{i} \tau_{i}}
$$

The mean lifetime of the excited state is given by:

$$
\bar{\tau}=\sum_{i} f_{i} \tau_{i}
$$

and the amplitude-weighted lifetime is given by:

$$
\langle\tau\rangle=\sum_{i} \alpha_{i} \tau_{i}
$$

The values of $\alpha_{i}$ and $\tau_{i}$ were determined by non-linear least squares impulse reconvolution with a goodness-offit $\chi^{2}{ }_{\mathrm{R}}$ criterion. The decay times are shown in Table 1 . The lifetimes decreased as the concentration of dextran was increased, which resulted in the aggregation of the nanoparticles, implying that the enhancement in the luminescence intensity (Figure 9) was due in part to the surface- enhanced fluorescence [30].
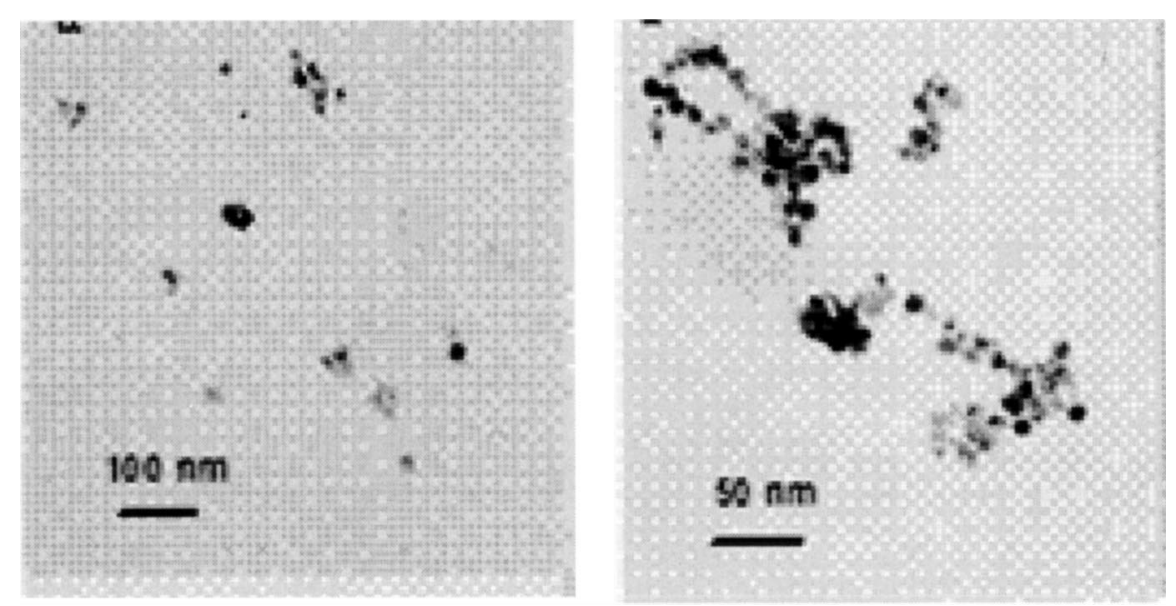

Fig. 8. Transmission electron micrograph of SNBA before (Top) and after the addition of $0.6 \mu \mathrm{M}$ dextran (Bottom). 

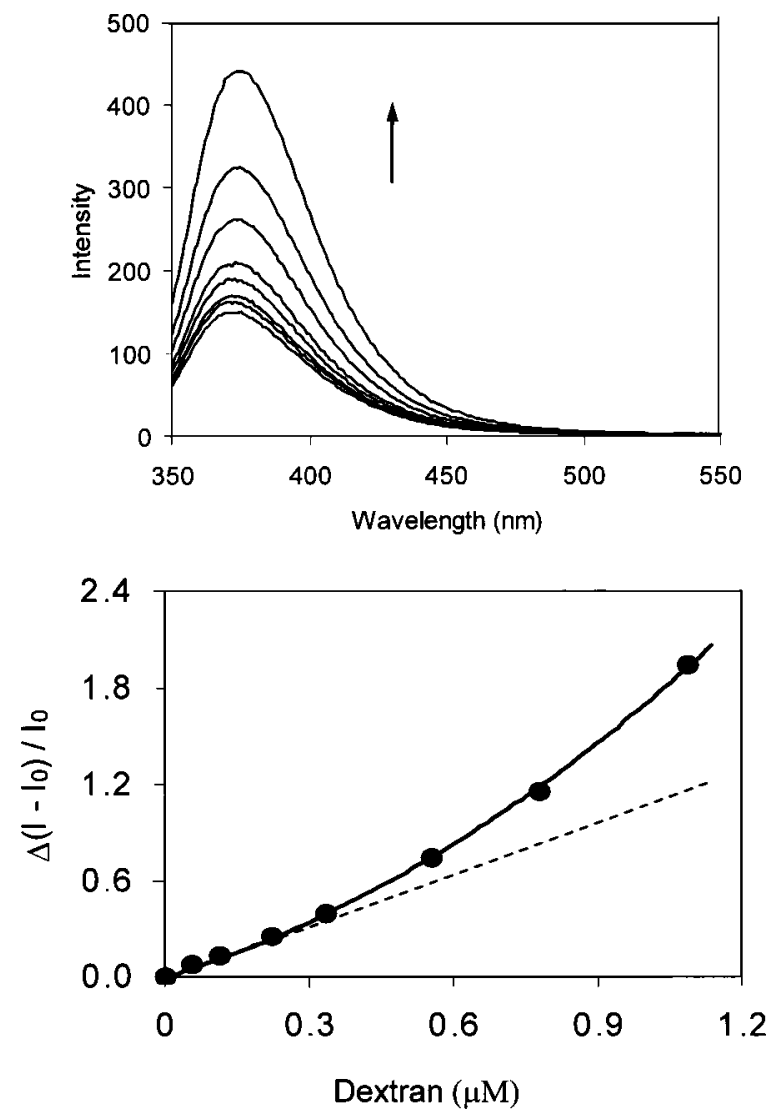

Fig. 9. Luminescence spectra of the SNBA before and after the addition of dextran (Top), luminescence intensity of SNBA at $375 \mathrm{~nm}$ vs. the concentration of dextran in water (Bottom).

\section{CONCLUSIONS}

We have reviewed some of our recently reported new methods for glucose sensing which utilize biomolecules,

Table I. Data Obtained Using the Multi-Exponential Model When the Concentration of SNBA was $60 \mathrm{nM}$ (Water)

\begin{tabular}{lccccc}
\hline \multicolumn{1}{c}{ Sample } & $\tau_{1,2}(\mathrm{~ns})$ & $\alpha_{1,2}$ & $\bar{\tau}(\mathrm{ns})$ & $\langle\tau\rangle(\mathrm{ns})$ & $\chi^{2}$ \\
\hline Compound 1 & $1.71(0.0950)$ & 0.2256 & 5.72 & 5.07 & 0.99 \\
& $6.05(0.0750)$ & 0.7744 & & & \\
SNBA & $1.47(0.0551)$ & 0.1459 & 4.95 & 4.59 & 1.07 \\
$(0 \mathrm{M}$ Dextran $)$ & $5.12(0.0734)$ & 0.8541 & & & \\
SNBA & $1.64(0.1682)$ & 0.2403 & 4.87 & 4.34 & 0.92 \\
$\left(1.1 \times 10^{-7}\right.$ M Dextran) & $5.19(0.0771)$ & 0.7599 & & & \\
SNBA & $1.42(0.0685)$ & 0.2233 & 4.93 & 4.36 & 0.90 \\
$\left(2.2 \times 10^{-7}\right.$ M Dextran) & $5.21(0.0435)$ & 0.7767 & & & \\
SNBA & $1.29(0.0573)$ & 0.2817 & 4.69 & 3.98 & 0.97 \\
$\left(3.3 \times 10^{-7}\right.$ M Dextran) & $5.03(0.0388)$ & 0.7183 & & & \\
SNBA & $1.05(0.0577)$ & 0.2460 & 4.53 & 3.86 & 1.08 \\
$\left(7.8 \times 10^{-7}\right.$ M Dextran) & $4.78(0.0405)$ & 0.7540 & & & \\
\hline
\end{tabular}

organic molecules and noble metal nanoparticles [19,22, 31-34]. Gold and silver nanoparticles were employed as glucose sensing platforms through the modification of their surfaces with dextran and boronic acid groups, respectively. The glucose sensing is based on the aggregation of these nanoparticles due to specific biological interactions and on the dissociation of the aggregates by competitive binding of glucose. Simple optical density and luminescence emission measurements were used as the primary measurement techniques for glucose sensing. Transmission Electron Microscopy was also used to provide qualitative assessment of the aggregation of nanosensors.

We believe that these new methods provide a framework for developing a cross-the-board technology for saccharide sensing in physiological fluids such as urine, blood and tears due their tunable sensitivity and physiologically compatible observation wavelengths.

\section{ACKNOWLEDGMENTS}

This work was supported by the National Center for Research Resources, RR-08119.

\section{REFERENCES}

1. M. R. Robinson, R. P. Eaton, D. M. Haaland, G. W. Koepp, E. V. Thomas, B. R. Stallard, and P. L. Robinson (1992). Clin. Chem. 38, 1618.

2. H. M. Heise, R. Marbach, T. H. Koschinsky, and F. A. Gries (1994). Ann. Occup. Hyg. 18, 439.

3. W. F. March, B. Rabinovitch, R. Adams, J. R. Wise, and M. Melton (1982) Trans. Am. Soc. Artif. Intern. Organs 28, 232.

4. B. Rabinovitch, W. F. March, and R. L. Adams (1982). Diabetes Care 5, 254.

5. G. M. Schier, R. G. Moses, I. E. T. Gan, and S. C. Blair (1988) Diabetes Res. Clin. Pract. 4, 177.

6. A. Labande and D. Astrue (2000). Colloids as redox sensors: Recognition of $\mathrm{H}_{2} \mathrm{PO}_{4}^{-}$and $\mathrm{HSO}_{4}^{-}$by amidoferrocenylalkylthiol-gold nanoparticles. Chem. Commun. 12, 1007-1008.

7. G. Schmid (1994). Clusters and Colloids from Theory to Applications, VCH, New York.

8. A. Henglein (1993). Physicochemical properties of small metal particles in solution: "Microelectrode" reactions, chemisorption, composite metal particles, and the atom-to-metal transition. J. Phys. Chem. 97, 5457-5471.

9. J. Belloni (1996). Curr. Opin. Colloid Interface Sci. 1, 184.

10. U. Kreibig and M. Vollmer (1995). Optical Properties of Metal Clusters, Springer, Berlin.

11. J. J. Storhoff, A. A. Lazarides, R. Mucic, C. A. Mirkin, R. Letsinger, and G. C. Schatz (2000). What controls the optical properties of DNA-linked gold nanoparticle assemblies. J. Am. Chem. Soc. 122, 4640-4650.

12. A. A. Lazarides and G. C. Schatz (2000). DNA-linked metal nanosphere materials: Structural basis for the optical properties. $J$. Phys Chem. B. 104, 460-467.

13. C. A. Mirkin and J. J. Storhoff (1999). Programmed materials synthesis with DNA. Chem. Rev. 99, 1849-1862. 
14. R. A. Reynolds, C. A. Mirkin, and R. L. Letsinger (2000). Homogeneous, nanoparticle-based quantitative colorimetric detection of oligonucleotides. J. Am. Chem. Soc. 122, 3795-3597.

15. C. A. Mirkin, R. L. Letsinger, R. L. Mucic, and J. J. Storhoff (1996). A DNA-based method for rationally assembling nanoparticles into macroscopic materials. Nature 382, 607-609.

16. Y. Kim, R. C. Johnson, and J. T. Hupp (2001). Gold nanoparticlebased sensing of "Spectroscopically Silent" heavy metal ions. Nano Lett. 1, 165-167.

17. S. Y. Lin, S. W. Liu, C. M. Lin, and C. H. Chen (2002). Recognition of potassium ion in water by 15 -crown-5 functionalized gold nanoparticles. Anal. Chem. 74, 330-335.

18. N. T. K. Thanh and Z. Rosenzweig (2002). Development of an aggregation-based immunoassay for anti-protein A using gold nanoparticles. Anal. Chem. 74, 1624-1628.

19. C. D. Geddes, K. Aslan, J. Zhang, and J. R. Lakowicz (in press). SPIE Proc.

20. S. Lofas and B. Johnsson (1990). A novel hydrogel matrix on gold surfaces in surface plasmon resonance sensors for fast and efficient covalent immobilization of ligands. J. Chem. Soc. Chem. Commun. $1526-1528$.

21. C. S. Weisbecker, M. G. Merritt, and G. M. Whitesides (1996). Molecular self-assembly of aliphatic thiols on gold colloids. Langmuir 12, 3763-3772.

22. J. Zhang, C. D. Geddes, and J. R. Lakowicz (in press). SPIE Proc.

23. K. Aslan and V. H. Pérez-Luna (2002). Surface modification of colloidal gold by chemisorption of alkane thiols in the presence of a nonionic surfactant. Langmuir 18, 6059-6065.

24. G. Springsteen, C. E. Ballard, S. Gao, W. Wang, and B. Wang, (2001). The development of photometric sensors for boronic acids. Bioorg. Chem. 29, 259-270.
25. A.-J. Tong, A. Yamauchi, T. Hayashita, Z.-Y. Zhang, B. D. Smith, and N. Teramae, (2001). Boronic acid fluorophore/beta-cyclodextrin complex sensors for selective sugar recognition in water. Anal. Chem. 73, 1530-1536.

26. J. R. Lakowiczc (2001). Radiative decay engineering: Biophysical and biomedical applications. Anal. BioChem. 298, 1-24.

27. J. R. Lakowicz, Y.B. Shen, S. D’Auria, J. Malicka, J.Y. Fang, Z. Gryczynski, and I. Gryczynski (2002). Radiative decay engineering 2. Effects of silver island films on fluorescence intensity, lifetimes, and resonance energy transfer. Anal. BioChem. 301, 261-277.

28. C. D. Geddes, H. Cao, I. Gryczynski, Z. Gryczynski, J. Y. Fang, and J. R. Lakowicz (2003). Metal-enhanced fluorescence (MEF) due to silver colloids on a planar surface: Potential applications of indocyanine green to in vivo imaging. J. Phys. Chem. A. 107, 3443-3449.

29. J. R. Lakowicz (1997). Principles of Fluorescence Spectroscopy, 2nd ed., Kluwer Academic/Plenum, New York.

30. J. Malicka, I. Gryczynski, and J. R. Lakowicz, (2003). Enhanced emission of highly labeled DNA oligomers near silver metallic surfaces. Anal. Chem. 75, 4408-4414.

31. K. Aslan, J. R. Lakowicz, and C. D. Geddes (in press). Plasmonic tunable glucose sensing based on the dissociation of Con A-aggregated dextran-coated gold colloids. Anal. Chim. Acta. (In press).

32. K. Aslan, J. R. Lakowicz, and C. D. Geddes (2004). Nanogold plasmon resonance based glucose sensing. Anal. Biochem. (Submitted).

33. J. Zhang, C. D. Geddes, and J. R. Lakowicz (2004). Competitive complexation of dextran and glucose with boronic acid capped on silver nanoparticles. Langmuir (Submitted).

34. J. Zhang, D. Roll, C. D. Geddes, and J. R. Lakowicz (2004). Aggregation of silver nanoparticle-dextran adducts with/without Concanavalin A and competitive displacement with glucose. J. Phys. Chem. B. (Submitted). 